\title{
Electromagnetic Driving Modal and Control of Magnetic Levitation Spherical Active Joint
}

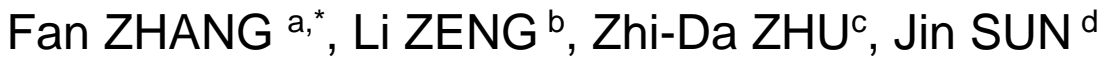 \\ College of Mechanical Engineering Yangzhou University, Yangzhou 225127, China \\ a fzhang@yzu.edu.cn, b lizengcf@163.com, c zdzhu@yzu.edu.cn, dinsun@yzu.edu.cn
}

Keywords: Magnetic levitation, Sphere active joint, States reconstruction, Control characteristic.

\begin{abstract}
The traditional universal driving joint has the friction and wear, so its moving parts would get hot and its dynamic performance would become worse when working. Based on the Integration of motors, magnetic levitation, robotics and other techniques, a novel magnetic levitation spherical reluctance active joint with multi-degree-of-freedom is proposed, which is a kind of mechatronical driving structure with compact structure and high integration of mechanical and electrical elements, which has advantages in control and trajectory planning. The distribution of air-gap magnetic conductance in the joint is derived with the magnetic field segmentation method, and the conversion principle model between electronical energy and mechanical energy of the joint's air gap magnetic-energy is established, and then the magnetic levitation force and magnetic torque produced by the joint are derived. The integrated control system is established for the joint system, and the simulation results show that this joint system has good dynamic and static performance.
\end{abstract}

\section{Introduction}

The traditional joints with multi-degree-of-freedom are usually composed of several single-degreeof-freedom joints which are connected by rods, and use multiple sets of the driving mechanism with single-degree-of-freedom and complex mechanical transmission mechanism to coordinate the translation and revolve of different degree-of-freedom[1-3]. Therefore, a joint with multi-degree-offreedom has some shortcomings, such as complex structure, large volume, serious wear on joint friction face, low efficiency, difficulty of manufacturing and assembling, slow response, and poor dynamic performance. And the cumulative error of mechanical transmission system would decrease the precision of the whole control system, and even would affect the stability of the whole system[4]. Since 1950s, because of the rapid development of robot, space technology and automation technology, the principle and structure research of spherical motor with multi-degreeof-freedom has much progress[5-7]. The magnetic levitation spherical reluctance active joint is also a kind of spherical active joint with multi-degree-of-freedom. This joint has a high level of mechanical integration, and can replace the multi-degree-of-freedom joint which has two or more single-degree-of-freedom transmission mechanism driven by motor and in a multi-degree-offreedom mechanical motion system, and then greatly simplifies the mechanical system structure and motion transmission chain. It also can reduce the volume and the weight, and eliminate the friction and backlash between the gear pair in a gear driving device. Thus it improves efficiency, precision and dynamic performance of the system and has an advantage in control and path planning, and the system would have high cost performance. But now the spherical motor generally has multilayer framework structures in order to achieve different rotational degrees of freedom, each of which is supported by mechanical bearings and can rotate or swing around a coordinate axis. So it has some disadvantages as complex structure, large volume, and limitation of rotation angle. When the multidegree-of-freedom joint device is working with ultra-high-speed or high-speed, the friction resistance of joints would be increased, that would cause parts hot, and the dynamic characteristics of joints become worse. Based on the magnetic levitation, robotics and motor technology, a novel multi-degree-of-freedom magnetic levitation spherical reluctance driving joint is proposed, which hasn't be reported. This joint suspends the spherical rotor by magnetic levitation force, and causes 
there no friction and no wear between stator and rotor, so has high system accuracy and good dynamic performance [7-10]. And then the working mechanism of magnetic suspension force and magnetic torque is studied, and the comprehensive control of magnetic torque is presented.

\section{The Structure Composition of Magnetic Levitation Spherical Reluctance Driving Joint}

Under the active control of the controller, the magnetic levitation spherical driving joint can produce the electromagnetic levitation force which is supporting for the joint rotor and the electromagnetic torque which drive the joint rotor rotating, it owns two or three rotating freedom and takes rotating work with non-contact and abrasion free bypass the fixed point of the space axes. It is mainly made up of several motor stators driving joint and the spherical driving joint rotor with the tumbler, etc., and the three-dimensional structure is shown in Fig. 1. The joint rotor is surrounded by the joints of the motor stator, the four stators are symmetrical distribution at the equatorial line position, the stator 1 and 3 are symmetry for spherical rotor and keep coaxial along the $\mathrm{X}$-axis, its effect is to drive the rotor rotating around the $\mathrm{X}$-axis and make rotor produce the stability magnetic levitation force in the $\mathrm{X}$-axis; the stator 2 and 4 are symmetry for spherical rotor and keep coaxial along the $\mathrm{Y}$-axis, its effect is to drive the rotor rotating around the $\mathrm{Y}$-axis and make rotor produce the stability magnetic levitation force in the Y-axis; the stator 5 arranges the top of spherical rotor and keeps coaxial with the Z-axis, its function is driven rotor spinning around the $\mathrm{Z}$-axis and make the rotor produce the stability magnetic levitation force in the Z-axis.

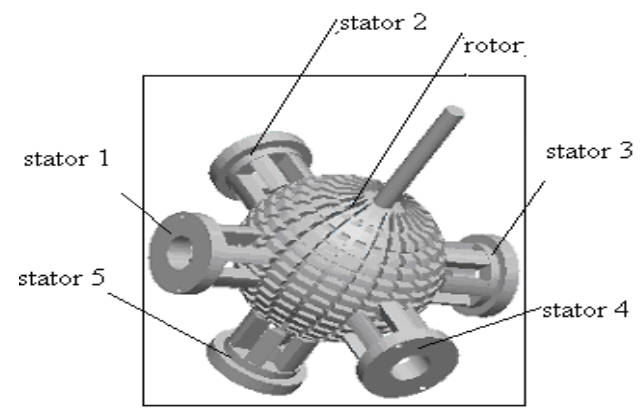

Fig.1. Structure of magnetic levitation spherical active joint

Some shapes of saliency column distributed in the outside surface of magnetic levitation spherical joint (cylinder or Prism Object), the capital envelope spherical surface is rotor spherical surface; The stator joint contains a number of similar saliency shape salient pole, and according to principle of reluctance motor, multi-phase $(\mathrm{m})$ winding winds around the salient pole which drives the rotor rotating, each phase windings have a pole. Each phase windings after electrify produce electromagnetic torque to drive the spherical rotor and also provides the radial levitation force for the spherical rotor.

\section{The Air-gap Magnetic Energy and Permeance of Magnetic Levitation Spherical Reluctance Driving joint}

Based on the spherical reluctance motor drive and support the spherical rotor with the tumbler, the magnetic levitation spherical reluctance driving joint realizes multi-freedom joints. Therefore, the study is the same of other analysis method of the reluctance, from the air-gap of the motor stator and rotor, the mechanical energy transformational relation is established, and then it get the relation between the electromagnetic levitation force which drives rotor suspending and electromagnetic torque which drives the rotor rotating. 


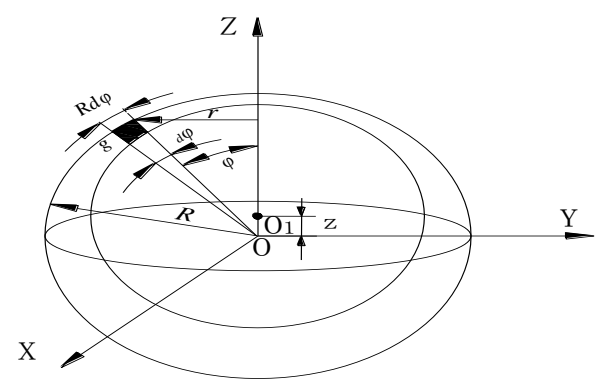

Fig.2. Schematic diagram of the rotor air gap

If the center of sphere of the magnetic spherical reluctance driving joint doesn't happen excursion, the radius of the stator salient pole envelope spherical surface is $\mathrm{R}$, the radius of the rotor salient pole envelope spherical surface is $\mathrm{Rr}$, the clearance between the stator salient pole envelope spherical surface and the rotor salient pole envelope spherical surface is $g 0=R-R r$. Make the symmetry axis of the stator to coincide with $Z$ axis and build frame of axes $f(X, Y, Z)=F(R, \varphi, \theta)$, as shown in Fig. 2. When the rotor joint is in the process of rotating, its salient pole surface is the overlap alignment with stator salient pole surface and it produces magnetic-pull (thrust) force to form electromagnetic torque, magnetic-pull force is the magnetic levitation force which drives the rotor suspend. Magnetic-pull (thrust) force is directly related to the size and distribution of the airgap magnetic conductance.

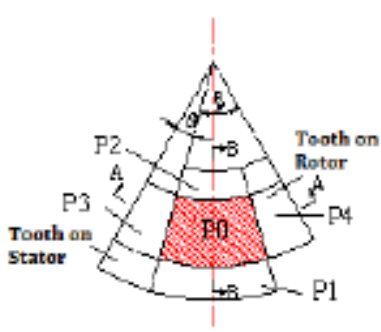

(a)the distribution of themagnetic conductance

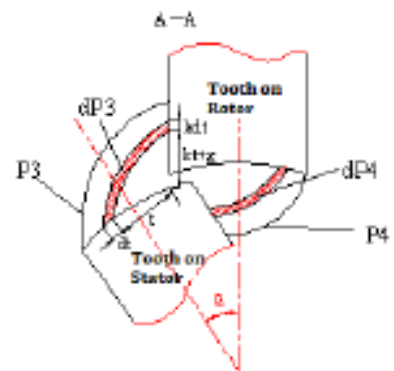

(b)the rotor rotating to causethe edgemagnetic conductance

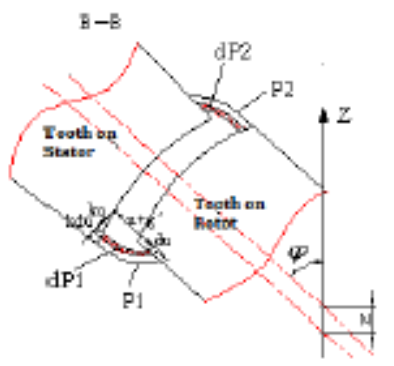

(c) the rotor offset to cause the edgemagnetic permeance

Fig.3. Magnetic paths partition of air gap

If the joint rotor is under the action of the interference, the centre-of-sphere of the rotor shifts from $\mathrm{O}$ to $\mathrm{O} 1$, the offset is $\mathrm{z}$ along the $\mathrm{Z}$-axis, when the angle is $\theta$ which rotated around the $\mathrm{Z}$-axis, the gear upper surface of the stator salient pole and the rotor salient pole will be stagger in the direction of the rotor offset (front, back) and the rotor rotating (left, right), it likes the Fig. 3. By using the method of the air-gap magnetic field segmentation to calculate magnetic conductance, it is divided into five parts, there are the magnetic conduction $\mathrm{P} 0$ of the overlap between the stator and the magnetic top of the rotor as shown in the Fig. 3(a), the edge magnetic (left flank and right flank) conduction P3. P4 which is a cause for the rotating rotor as shown in the Fig. 3(b); the edge magnetic (front flank and back flank) conduction P1. P2 which is a cause for the offset of the rotor 
as shown in the Fig. 3(c). The total magnetic conduction of this mutual interaction between the airgap magnetic is:

$$
P(z, \theta)=P_{0}+P_{1}+P_{2}+P_{3}+P_{4}
$$

Because the departure of the rotor from the equilibrium position is very small, the surface between the stator and the magnetic the rotor salient pole is considered the parallel surface, the magnetic circuit of the overlap between the stator gear and the rotor gear can be instead of the linear magnetic circuit; the elliptic curve approximately replaces the edge magnetic flow path[11]. After simplifying like this, the calculation of the total magnetic conduction of the interaction between the pair poles for air-gap is:

$$
\begin{aligned}
& P(z, \theta)=\frac{\mu_{0}(L-r \theta)(L-z \sin \varphi)}{g}+\frac{2 \mu_{0}}{\pi}(L-r \theta) \ln \frac{2 z^{2} \sin ^{2} \varphi+2 g z \sin \varphi+g^{2}}{g^{2}} \\
& +\frac{2 \mu_{0}}{\pi}(L-z \sin \varphi) \ln \frac{g^{2}+2 g r \theta+2 r^{2} \theta^{2}}{g^{2}}
\end{aligned}
$$

Where $\mu 0$ is the space permeability, $\mathrm{r}$ is the inside circular arc radius of the stator magnetic pole, $\mathrm{L}$ is the side length of the stator tooth notching, $\varphi$ indicates the angle between the position of the stator tooth shape at normal and Z-axis, $g=g_{0} \mp z \cos \varphi$ is the radial air gap length between the crest top land of the rotor salient pole and the crest top land of the stator salient pole (magnetic pole), when the rotor near the stator, to take "-", when the rotor depart from the stator, to take "+", g0 is the air-gap radius between the magnetic pole and the stator magnetic pole when the rotor doesn't have offset.

If the stator motor joint has $\mathrm{m}$ phase windings, each phase winding has a magnetic pole to work with electricity, according to the theory of motor, the calculation of the total energy which is stored in the air-gap magnetic field of the joint stator motor is:

$$
W_{g}=\frac{1}{2} L m i^{2}=\frac{1}{2} K m N^{2} i^{2} P(z, \theta)
$$

Where $\mathrm{N}$ is turns per coil of onephase winding, $L=K N^{2} P(z, \theta)$ is the inductance which onephase winding generates.

When the spherical rotor makes the offset along X-axis or Y-axis, similarly it can deduce the magnetic conduction of the joint air gap and the total magnetic energy which is the same structure of the (2) and the (3). According to the magnetic energy Wg of the joint air gap, the magnetic levitation force which the joint generates can be taken by making the derivation of the offset of the rotor along the coordinate, the electromagnetic torque of the driving rotor which the joint generates can be taken by making the derivation of the rotor angle $\theta$.

\section{The Magnetic Levitation Force and Electromagnetic Torque of Magnetic Levitation Spherical Reluctance Driving Joint}

According to (3), it can take the magnetic levitation force and the electromagnetic torque of the magnetic levitation spherical reluctance driving joint along the Z-axis by making the partial derivative of $\mathrm{z}$ and $\theta$ : 


$$
\left\{\begin{array}{l}
F_{z}=\frac{1}{2} K m N^{2} i^{2}\left[\frac{\mu_{0}(L-r \theta)[g(-\sin \varphi)-(L-z \sin \varphi)(\mp \cos \varphi)]}{g^{2}}+\right. \\
\frac{4 \mu_{0}(L-r \theta)(2 z \sin \varphi+g) g_{0} \sin \varphi}{\pi g\left(2 z^{2} \sin ^{2} \varphi+2 g z \sin \varphi+g^{2}\right)}-\frac{2 \mu_{0} \sin \varphi}{\pi} \ln \frac{g^{2}+2 g r \theta+2 r^{2} \theta^{2}}{g^{2}} \\
\left.+\frac{\left.4 \mu_{0} r \theta(L-z \sin \varphi)(g+2 r \theta)(\mp \cos \varphi)\right]}{\pi g^{3}}\right] \\
\quad T_{z}=\frac{1}{2} K m N^{2} i^{2}\left[\frac{4 \mu_{0} r(L-z \sin \varphi)(g+2 r \theta)}{\pi\left(g^{2}+2 g r \theta+2 r^{2} \theta^{2}\right)}-\frac{\mu_{0} r(L-z \sin \varphi)}{g}-\right. \\
\left.\frac{2 \mu_{0} r}{\pi} \ln \frac{2 z^{2} \sin ^{2} \varphi+2 g z \sin \varphi+g^{2}}{g^{2}}\right]
\end{array}\right.
$$

According to (4), through controlling the supply current of the joints, it can adjust the size of magnetic levitation force and the electromagnetic torque.

For the $\mathrm{X}$-axis (Y-axis) direction, due to the two stator electromagnet driving joint rotor to move, so its magnetic levitation force is:

$$
\begin{aligned}
& F_{x}=F_{x 1}-F_{x 2} \\
& =\frac{1}{2} K m N^{2} i_{2}^{2}\left[\frac{\mu_{0}(L-r \theta)\left[g_{x 1}(-\sin \varphi)-(L-x \sin \varphi) \cos \varphi\right]}{g_{x 1}^{2}}+\right. \\
& \frac{4 \mu_{0}(L-r \theta)\left(2 x \sin \varphi+g_{x 1}\right) g_{0} \sin \varphi}{\pi g_{x 1}\left(2 x^{2} \sin ^{2} \varphi+2 g_{x 1} x \sin \varphi+g_{x 1}^{2}\right)}-\frac{2 \mu_{0} \sin \varphi}{\pi} \ln \frac{g_{x 1}^{2}+2 g_{x 1} r \theta+2 r^{2} \theta^{2}}{g_{x 1}^{2}} \\
& \left.+\frac{\left.4 \mu_{0} r \theta(L-x \sin \varphi)\left(g_{x 1}+2 r \theta\right) \cos \varphi\right]}{\pi g_{x 1}^{3}}\right]- \\
& \frac{1}{2} K m N^{2} i_{2}^{2}\left[\frac{\mu_{0}(L-r \theta)\left[g_{x 2}(-\sin \varphi)-(L-x \sin \varphi) \cos \varphi\right]}{g_{x 2}^{2}}+\right. \\
& \frac{4 \mu_{0}(L-r \theta)\left(2 x \sin \varphi+g_{x 2}\right) g_{0} \sin \varphi}{\pi g_{x 2}\left(2 x^{2} \sin ^{2} \varphi+2 g_{x 2} X \sin \varphi+g_{x 2}^{2}\right)}-\frac{2 \mu_{0} \sin \varphi}{\pi} \ln \frac{g_{x 2}^{2}+2 g_{x 2} r \theta+2 r^{2} \theta^{2}}{g_{x 2}^{2}} \\
& \left.+\frac{\left.4 \mu_{0} r \theta(L-x \sin \varphi)\left(g_{x 2}+2 r \theta\right) \cos \varphi\right]}{\pi g_{x 2}^{3}}\right]
\end{aligned}
$$

The air gap between the two stator and rotor magnetic poles is: $g_{x 1}=g_{0}-x \cos \varphi, g_{x 2}=g_{0}+x \cos \varphi$. Similarly the total electromagnetic torque of the two joints stator which drives the spherical rotor rotating is:

$$
\begin{aligned}
& T_{x}=T_{x 1}+T_{x 2} \\
& =\frac{1}{2} \cdot K m N^{2} i^{2}\left[\frac{4 \mu_{0} r(L-x \sin \varphi)\left(g_{x 1}+2 r \theta\right)}{\pi\left(g_{x 1}^{2}+2 g_{x 1} r+2 r^{2} \theta^{2}\right)}-\frac{\mu_{0} r(L-x \sin \varphi)}{g_{x 1}}-\right. \\
& \left.\frac{2 \mu_{0} r}{\pi} \ln \frac{2 x^{2} \sin ^{2} \varphi+2 g_{x 1} z \sin \varphi+g_{x 1}^{2}}{g_{x 1}^{2}}\right]+\frac{1}{2} K m N^{2} i^{2}\left[-\frac{\mu_{0} r(L-x \sin \varphi)}{g_{x 1}}-\right. \\
& \left.\frac{2 \mu_{0} r}{\pi} \ln \frac{2 x^{2} \sin ^{2} \varphi+2 g_{x 1} z \sin \varphi+g_{x 1}^{2}}{g_{x 1}^{2}}+\frac{4 \mu_{0} r(L-x \sin \varphi)\left(g_{x 1}+2 r \theta\right)}{\pi\left(g_{x 1}^{2}+2 g_{x 1} r \theta+2 r^{2} \theta^{2}\right)}\right]
\end{aligned}
$$

According the (5) and (6), the magnetic levitation force and electromagnetic torque mutually interference and influence which the joint generates, the whole system is the nonlinear strong coupled system and needs nonlinear decoupling control.

\section{The Inverse System Model of Magnetic Levitation Spherical Reluctance Driving Joint}

According to Newton's second law, the equation of the spherical rotor moving along the $\mathrm{X}$ axis and rotating around the $\mathrm{X}$ axis is

$$
\left\{\begin{array}{l}
M \frac{d^{2} x}{d t^{2}}=F_{x}+F_{d}=\frac{i_{1}^{2} K_{f}(\psi)}{2 g_{x 1}^{2}}-\frac{i_{2}^{2} K_{f}(\psi)}{2 g_{x 2}^{2}}+F_{x} \\
J \frac{d^{2} x}{d t^{2}}=M_{x}+M_{d}=\frac{i_{1}^{2} K_{m}(\psi)}{2 g_{x 1}^{2}}-\frac{i_{2}^{2} K_{m}(\psi)}{2 g_{x 2}^{2}}+M_{x}
\end{array}\right.
$$


Where M, J --The mass and rotary inertia of the spherical rotor; Fd, Md -- the disturbance force and torque of the spherical rotor beside the electromagnetic suspension force and torque.

Then the relationship between the current $i$ and the voltage $u$ in the joint winding is obtained by the law of electromagnetic induction.

$$
\left\{\begin{array}{l}
u_{1}=R i_{1}+L_{x 1} \frac{d i_{1}}{d t}+i_{1}\left(\frac{\partial L_{x 1}}{\partial x} \frac{d x}{d t}+\frac{\partial L_{x 1}}{\partial \psi} \frac{d \psi}{d t}\right) \\
u_{2}=R i_{2}+L_{x 2} \frac{d i_{2}}{d t}+i_{2}\left(\frac{\partial L_{x 2}}{\partial x} \frac{d x}{d t}+\frac{\partial L_{x 2}}{\partial \psi} \frac{d \psi}{d t}\right)
\end{array}\right.
$$

Where $\left\{\begin{array}{l}\frac{\partial L_{x 1}}{\partial x}=\frac{K_{f}(\psi)}{g_{x 1}^{2}} \\ \frac{\partial L_{x 1}}{\partial \psi}=\frac{K_{m}(\psi)}{g_{x 1}}\end{array},\left\{\begin{array}{l}\frac{\partial L_{x 2}}{\partial x}=\frac{K_{f}(\psi)}{g_{x 2}^{2}} \\ \frac{\partial L_{x 2}}{\partial \psi}=\frac{K_{m}(\psi)}{g_{x 2}}\end{array}\right.\right.$,

$$
\begin{aligned}
K_{f}(\psi)= & \frac{1}{2} K m N^{2}\left[\mu_{0}(L-r \theta)\left(-g_{x 1} \sin \varphi+L \cos \varphi-x \sin \varphi \cos \varphi\right)\right. \\
& +\frac{4 \mu_{0}(L-r \theta)\left(2 x \sin \varphi+g_{x 1}\right) g_{x 1} g_{0} \sin \varphi}{\pi\left(2 x^{2} \sin ^{2} \varphi+2 g_{x 1} x \sin \varphi+g_{x 1}^{2}\right)} \\
& -\frac{2 \mu_{0} g_{x 1}^{2} \sin \varphi}{\pi} \ln \frac{g_{x 1}^{2}+2 g_{x 1} r \theta+2 r^{2} \theta^{2}}{g_{x 1}^{2}} \\
& \left.-\frac{4 \mu_{0} r \theta(L-x \sin \varphi)\left(g_{x 1}+2 r \theta\right) \cos \varphi}{\pi g_{x 1}}\right]
\end{aligned}
$$

and

$$
\begin{aligned}
K_{m}(\psi) & =\frac{1}{2} K m N^{2}\left[\frac{4 \mu_{0} r(L-x \sin \varphi) g_{x 1}^{2}\left(g_{x 1}+2 r \theta\right)}{\pi\left(g_{x 1}^{2}+2 g_{x 1} r \theta+2 r^{2} \theta^{2}\right)}-\frac{\mu_{0} r g_{x 1}(L-x \sin \varphi)}{\pi}\right. \\
& \left.-\frac{2 \mu_{0} r g_{x 1}^{2}}{\pi} \ln \frac{2 x^{2} \sin ^{2} \varphi+2 g_{x 1} x \sin \varphi+g_{x 1}^{2}}{g_{x 1}^{2}}\right]
\end{aligned}
$$

In order to realize the decoupling control and linearization of magnetic levitation spherical reluctance driving joint system, the state space model of joint system is transformed from its mathematical model of system dynamics. Assuming the input variable vector is $\boldsymbol{U}=\left(\begin{array}{lll}u_{1} & u_{2}\end{array}\right)^{T}$, the

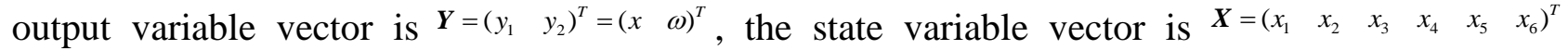
$=\left(\begin{array}{llllll}x & \dot{x} & \theta & \omega & i_{1} & i_{2}\end{array}\right)^{T}$, then the system state equations are

$$
\left\{\begin{array}{l}
\dot{x}_{1}=x_{2} \\
\dot{x}_{2}=\frac{1}{M}\left[\frac{K_{f}\left(x_{3}\right) x_{5}^{2}}{2 g_{x 1}^{2}}-\frac{K_{f}\left(x_{3}\right) x_{6}^{2}}{2 g_{x 2}^{2}}+F_{d}\right] \\
\dot{x}_{3}=x_{4} \\
\dot{x}_{4}=\frac{1}{J}\left[\frac{K_{m}\left(x_{3}\right) x_{5}^{2}}{g_{x 1}^{2}}-\frac{K_{m}\left(x_{3}\right) x_{6}^{2}}{g_{x 2}^{2}}+M_{d}\right] \\
\dot{x}_{5}=-\frac{R x_{5}}{L_{x 1}}-\frac{x_{5}}{L_{x 1}}\left(\frac{K_{f}\left(x_{3}\right)}{g_{x 1}^{2}} x_{2}+\frac{K_{m}\left(x_{3}\right)}{g_{x 1}} x_{4}\right)+\frac{u_{1}}{L_{x 1}} \\
\dot{x}_{6}=-\frac{R x_{6}}{L_{x 2}}-\frac{x_{6}}{L_{x 2}}\left(-\frac{K_{f}\left(x_{3}\right)}{g_{x 2}^{2}} x_{2}+\frac{K_{m}\left(x_{3}\right)}{g_{x 2}} x_{4}\right)+\frac{u_{2}}{L_{x 2}}
\end{array}\right.
$$

Equation 9 describes the dynamic coupling and nonlinear original system model of magnetic levitation spherical reluctance driving joint system in $\mathrm{X}$-axis direction. According to this prototype model, a decoupling control approach based on the inverse system model can be used on the joint system. 
Assuming the input variable vector is $\boldsymbol{A}(\boldsymbol{U})=\left(\begin{array}{lll}\ddot{y}_{1} & \dddot{y}_{2}\end{array}\right)^{T}=\left(\begin{array}{lll}\varphi_{1} & \varphi_{2}\end{array}\right)^{T}+\left(\begin{array}{ll}\frac{1}{M} \dot{F}_{d} & \frac{1}{J} \dot{M}_{d}\end{array}\right)^{T}$, according to the inverse algorithm of controller presented in literature [11], the Inverse of (9) is derived. The input signal of the inverse system of magnetic levitation spherical reluctance driving joint is

$$
\left\{\begin{aligned}
\varphi_{1} & =\frac{1}{M}\left[\frac{K_{f}\left(x_{3}\right) x_{5} \dot{x}_{5}}{g_{x 1}^{2}}-\frac{K_{f}\left(x_{3}\right) x_{6} \dot{x}_{6}}{g_{x 2}^{2}}\right. \\
& +\frac{x_{5}^{2} \dot{K}_{f}\left(x_{3}\right) g_{x 1}+2 x_{2} x_{5}^{2} K_{f}\left(x_{3}\right) \cos \varphi_{0}}{2 g_{x 1}^{3}} \\
& \left.-\frac{x_{6}^{2} \dot{K}_{f}\left(x_{3}\right) g_{x 2}+2 x_{2} x_{6}^{2} K_{f}\left(x_{3}\right) \cos \varphi_{0}}{2 g_{x 2}^{3}}\right] \\
\varphi_{2} & =\frac{1}{J}\left[\frac{K_{m}\left(x_{3}\right) x_{5} \dot{x}_{5}}{g_{x 1}}+\frac{K_{m}\left(x_{3}\right) x_{6} \dot{x}_{6}}{g_{x 2}}\right. \\
& +\frac{x_{5}^{2} \dot{K}_{m}\left(x_{3}\right) g_{x 1}+x_{2} x_{5}^{2} K_{m}\left(x_{3}\right) \cos \varphi_{0}}{g_{x 1}^{2}} \\
& \left.+\frac{x_{6}^{2} \dot{K}_{m}\left(x_{3}\right) g_{x 2}+x_{2} x_{6}^{2} K_{m}\left(x_{3}\right) \cos \varphi_{0}}{g_{x 2}^{2}}\right]
\end{aligned}\right.
$$

The combined solution of (10) is gotten as (11):

$$
\left\{\begin{array}{l}
\dot{x}_{5}=\frac{\left(g_{0}^{2}-x_{1}^{2} \cos \varphi_{0}\right)^{2}\left(M D \varphi_{1}+J B \varphi_{2}-B F-D E\right)}{2 g_{0} K_{f}\left(x_{3}\right) K_{m}\left(x_{3}\right) x_{5} x_{6}} \\
\dot{x}_{6}=\frac{\left(g_{0}^{2}-x_{1}^{2} \cos \varphi_{0}\right)^{2}\left(-M C \varphi_{1}+J A \varphi_{2}-A F-C E\right)}{2 g_{0} K_{f}\left(x_{3}\right) K_{m}\left(x_{3}\right) x_{5} x_{6}}
\end{array}\right.
$$

Where $A=\frac{K_{f}\left(x_{3}\right) x_{5}}{g_{x 1}^{2}}, B=\frac{K_{f}\left(x_{3}\right) x_{6}}{g_{x 2}^{2}}, C=\frac{K_{m}\left(x_{3}\right) x_{5}}{g_{x 1}}, D=\frac{K_{m}\left(x_{3}\right) x_{6}}{g_{x 2}}$,

$$
\begin{array}{r}
E=\frac{x_{5}^{2} \dot{K}_{f}\left(x_{3}\right) g_{x 1}+2 x_{2} x_{5}^{2} K_{f}\left(x_{3}\right) \cos \varphi_{0}}{2 g_{x 1}^{3}} \\
-\frac{x_{6}^{2} \dot{K}_{f}\left(x_{3}\right) g_{x 2}+2 x_{2} x_{6}^{2} K_{f}\left(x_{3}\right) \cos \varphi_{0}}{2 g_{x 2}^{3}}
\end{array}
$$

and

$$
\begin{aligned}
F & =\frac{x_{5}^{2} \dot{K}_{m}\left(x_{3}\right) g_{x 1}+x_{2} x_{5}^{2} K_{m}\left(x_{3}\right) \cos \varphi_{0}}{g_{x 1}^{2}} \\
& +\frac{x_{6}^{2} \dot{K}_{m}\left(x_{3}\right) g_{x 2}+x_{2} x_{6}^{2} K_{m}\left(x_{3}\right) \cos \varphi_{0}}{g_{x 2}^{2}} .
\end{aligned}
$$

So the output signal of the inverse system (the input signal of the original system) can be obtained from the state equation (9), which is shown in (12).

$$
\left\{\begin{array}{l}
u_{1}=L_{x 1} \dot{x}_{5}+R x_{5}+x_{5}\left(\frac{K_{f}\left(x_{3}\right) x_{2}}{g_{x 1}^{2}}+\frac{K_{m}\left(x_{3}\right) x_{4}}{g_{x 1}}\right) \\
u_{2}=L_{x 2} \dot{x}_{6}+R x_{6}+x_{6}\left(-\frac{K_{f}\left(x_{3}\right) x_{2}}{g_{x 2}^{2}}+\frac{K_{m}\left(x_{3}\right) x_{4}}{g_{x 2}}\right)
\end{array}\right.
$$

Equation (10) and (12) constitute the magnetic suspension spherical reluctance active joint system to $\varphi 1, \varphi 2$ as input, u1, u2 output for the alpha order integral inverse system mathematical model, as well as the design of algorithm formula of inverse system decoupling controller. 


\section{The Control System and Simulation Analysis of Magnetic Levitation Spherical Reluctance Driving Joint}

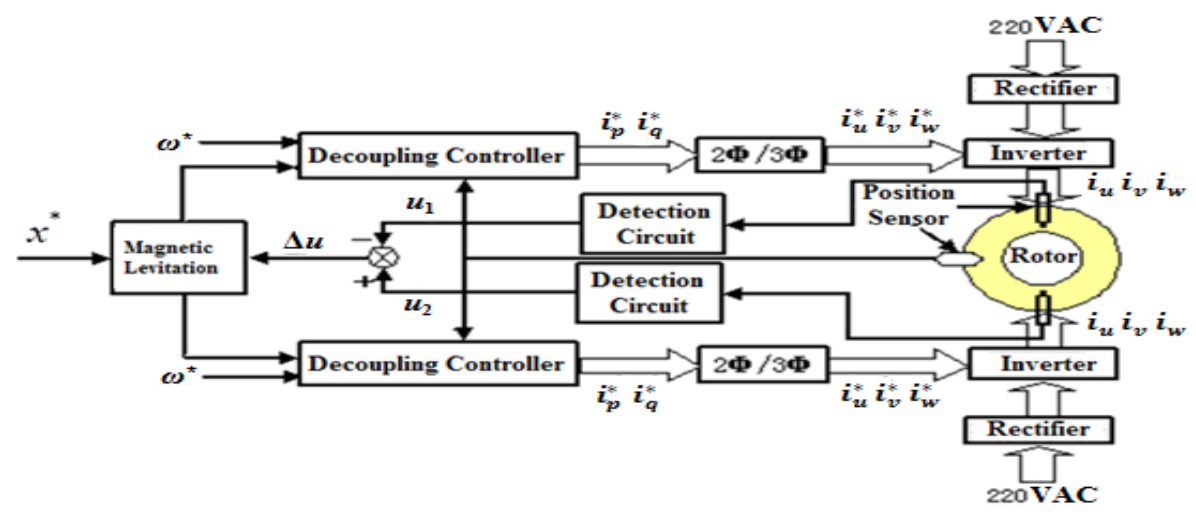

Fig.4. Control principle of spherical driving joint with magnetic levitation

Without the interference from the outside, equation (10) can be linearly decoupled and then a pseudo-linear system model for the rotor suspension and rotation of magnetic levitation spherical reluctance driving joint is gotten. Each equation gotten from decoupling represents an independent system, which is a part of the pseudo-linear system whose input variables are $\varphi 1$ and $\varphi 2$ and whose output variables are $\mathrm{x}$ and $\omega$. The characteristic roots of the pseudo-linear system are all zero, so after decoupling the controls of radial displacement and rotational speed of the rotor system are all unstable, and a closed loop comprehensive control must be carried out for achieving the stable suspension and rotation of the rotor.

In the same coordinate direction, two reluctance motor stator windings of magnetic levitation spherical reluctance driving joints can be alternative controlled according to the control strategy of the reluctance motor, the subsystem which constitutes two reluctance motor can synchronous drive sphere rotor rotating. Take two of the three-phase $(\mathrm{m}=3)$ motor driving in the $\mathrm{X}$-axis for example, It detects the offset signal of the rotor by displacement sensor, to convert it into current (or voltage signals) by after filtering and amplification and suspension controller, etc, after making feedback to the input of the windings control and comparing it with the displacement of the input signals, then to go through decoupling controller decoupling with the rotate speed signals of the rotor and transform the decoupling signal into the control signals of the three-phase circuit by $2 \Phi / 3 \Phi$, so as to change the current size of the stator winding and achieve suspension control and rotating control, the principle of control system is shown in Fig. 4.

According to the voltage equation of the joint stator winding and the kinematics equations of the spherical rotor, the nonlinear coupling dynamic mathematical model of magnetic levitation spherical reluctance driving joint, as shown in the Fig. 4, the control system begins to emulate magnetic levitation spherical reluctance driving joint and then can analyze the performance of the system.

(1) The simulation results of spherical rotor suspending

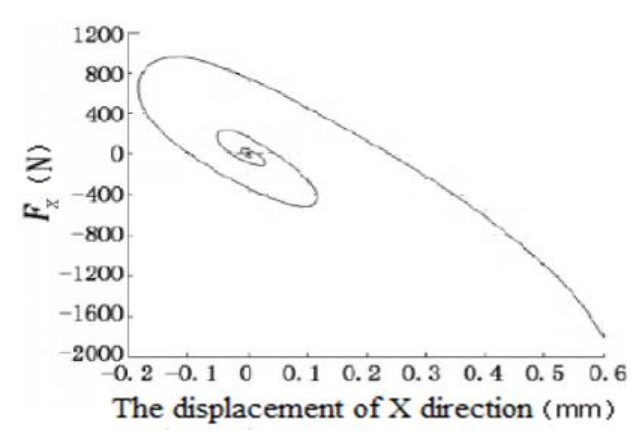

Fig.5. The relationship diagram between Electricallevitation force and axial displacement 
The relationship between the radial suspension force and the radial displacement of magnetic levitation spherical reluctance driving joint is shown in Fig. 5. While the rotor starts working, the radial displacement of rotor should be maxim. In order to restore the rotor to the balance position, the rotor should be pushed by the largest radial suspension force at this time. While the rotor reached the balance position, the rotor cannot stop immediately at just the right place because of the inertia of rotor and continues to move forward a little distance. Then the rotor should be pushed by a force in the opposite direction. After so many times, the spherical rotor should be slightly oscillating at the balance position, and the radial suspension force should change in a very small range. This state can be considered to achieve stable.

After the magnetic suspension spherical reluctance active joint starts to work, the rotor should quickly float from the initial position, then rapidly stabilize at the equilibrium position. As shown in Fig. 6, the rise time of this process is less than 0.3 seconds, and the overshoot is controlled less than $1 \%$, and the steady state error of this process is 0 , so the system has good dynamic and static performance.

Fig. 7 shows the displacement of the center of spherical rotor deviating from the balance position from 0.2 seconds to 1.4 seconds, after the joint rotor is basically stable with the $50 \mathrm{~Hz} \mathrm{AC}$ sine input signal. It can be seen from the figure that after the joint rotor position has be basically stable, the radial displacement of rotor should be less than $50 \mu \mathrm{m}$. Therefore, it is considered that the rotor is at the balance position and is stable.

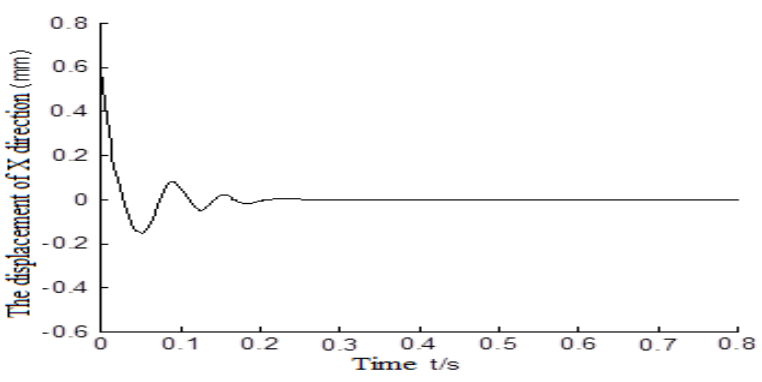

Fig.6. Radial levitation simulation in start-up process

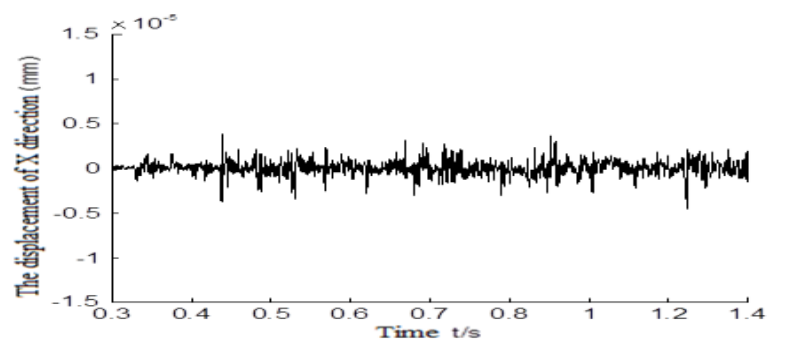

Fig.7. Radial levitation simulation in start-up process

(2)The simulation results of the joint rotor which is disturbed

After the driving joints achieved stabilize suspension, the simulation result of impulse interference in the positive and negative direction is shown in Fig. 8 which the joint rotor input when it is at 0.5 seconds and 0.8 seconds. According to the figure, if the magnetic levitation spherical reluctance driving joint received the radial disturbance, it can restore to the equilibrium position in a short period of time, the rising time is all under 0.2 seconds and the overshoot is small; it doesn't have the shocking phenomenon.

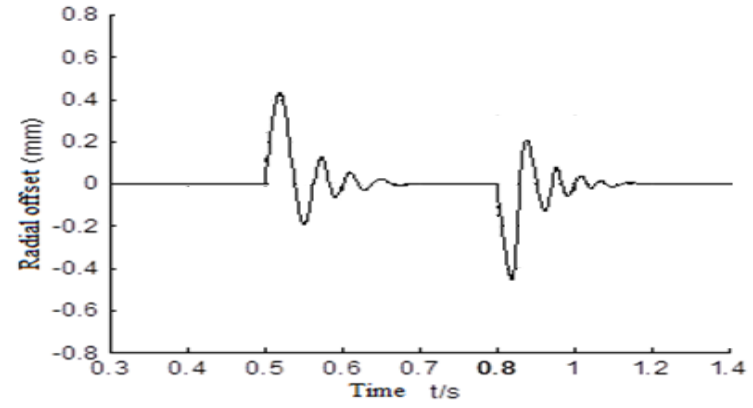

Fig. 8. Radial offset with interference of Laplace domain signals

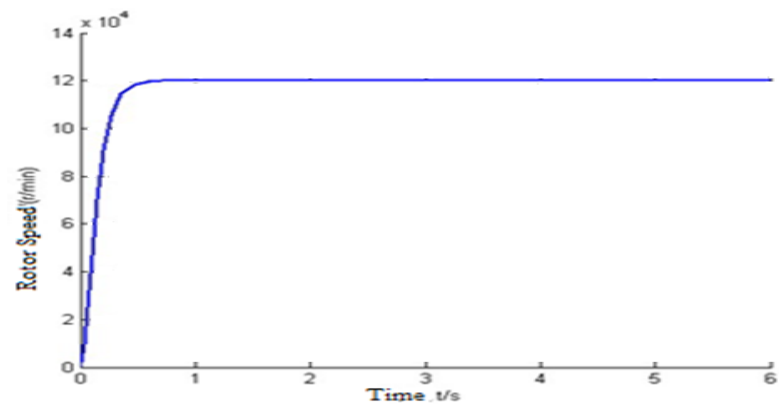

Fig.9. The dynamic response of the rotor speed

(3) The rotating speed response of the spherical rotor

After the inverse system decoupling and comprehensive correction, Fig. 9 shows the rotating speed (angular velocity) step response of the spherical rotor spinning around the X-axis, the expected rotating speed is $1.2 \times 105 \mathrm{r} / \mathrm{min}$. According to the results of the simulation and the rotating 
has less overshoot, the settling time is $0.5 \mathrm{~s}$, the subsystem of the rotating speed has good performance index

\section{Conclusions}

This paper presents the multi-degree-of-freedom magnetic levitation spherical reluctance driving joint which owns the simple structure, high precision and good dynamic performance; According to the air-gap magnetic energy, it established the transformational relation of the joint mechanical energy and studies the mechanism of the joint to produce electric magnetic levitation force and electromagnetic torque; according to this, the comprehensive control system of the joint is established and it made the system simulation analysis of it, the simulation results show that the driving joint system has good dynamic and static performance and the anti-interference ability.

\section{Acknowledgements}

Thank the China Natural Science Foundation for this project. Item Number: 51375427, 51475409. Thank the Jiang Su Natural Science Foundation for this project. Item Number: BK20131232, BK20141277. Thank the Jiang Su Natural Science Foundation for this project. Item Number: BY2014117-08, BY2015061-04.

\section{References}

1. Wang Guangjian, Liang Xichang, and Jiang Jiandong, The Present State and Developing Tendency of Robot Joint, Journal of Mechanical Transmission, vol.28, No.4, pp.1-5,2004.

2. Shirai Takeki. Joint Structure, Japan, JP2002113681, 2002

3. Xianwen Kong, Yan Jin, Type Synthesis of 3-DOF Multi-Mode Translational/Spherical Parallel Mechanisms with Lockable Joints Original Research, Mechanism and Machine Theory, Vol.96, Part 2, pp.323-333, February 2016.

4. Wu Junfei, Zhou Guilian, and Fu Ping, Research Progress of Drives Used in Robot Joint, Journal of Qingdao Institute of Chemical Technology, vol.23, No.3, pp.54-58, 2002.

5. Davey K, Vachtsevanos G, and Powers R, The analysis of fields and torques in spherical induction motors, IEEE Trans. on Magn, vol.23, No.1, pp.273-282,1987.

6. Kaneko K, Yamada I, and Itao K.,A spherical DC servo motor with three degrees of freedom, ASME Trans. Dynamic Systems Measurement and Control, vol.111, No.3, pp.398-402,1988.

7. Zeng Li, Wang Jun, and Dai min,Mechanism and control strategy of active magnetic levitation ball joint, Journal of Xuzhou Institute of Technology (natural science edition), No. 6, pp.1-5, 2010.

8. Zeng Li, Zhang Dan, and Dai Min,Maglev Spherical Reluctance Motor with Centripetal Thrust/Pull, China, ZL200920039032.7, 2010.

9. Zeng Li, Zhang Dan, and Jiang Mingming,Switch Reluctance Driving Joint with Magnetic Levitation,China, ZL200920039031.2, 2010.

10. M. Takemoto, A. Chiba, T. Fukao,A Feed-forward Compensator for Vibration Reduction Considering Magnetic Attraction Force in Bearingless Switched Reluctance Motors, Seventh International Sympsium on Magnetic Bearings. ETH Zurich,2000

11. Zhang Yuan, Research and Development of Bearingless Switched Reluctance Motor, Nanjing University of Aeronautics and Astronautics, pp.13-14,2005. 